\subsection{0}

doi: 10.15789/2220-7619-2018-4-6.10

GENOMIC EPIDEMIOLOGY OF TUBERCULOSIS: FROM WITHIN HOST EVOLUTION TO GLOBAL MIGRATION PATTERNS

I. Comas ${ }^{1}$, I. Cancino-Muñoz ${ }^{2}$, G.A. Goig ${ }^{1}$, Á. Chiner-Oms ${ }^{3}$, M.G. López ${ }^{1}$, M. Torres-Puente ${ }^{1}$, M.Á. Moreno-Molina ${ }^{1}$, L.M. Villamayor ${ }^{1}$, V. Furió ${ }^{1}$

${ }^{1}$ Tuberculosis Genomics Unit, Biomedicine Institute of Valencia, Spanish Research Council, Valencia, Spain; ${ }^{2}$ FISABIO Public Health, Valencia, Spain; ${ }^{3}$ Unidad Mixta Genómica y Salud, Centro Superior de Investigación en Salud Pública (FISABIO)-Universitat de València, Valencia, Spain

The availability of thousands of Mycobacterium tuberculosis genomes allows not only to infer how the pathogen emerged and spread but also to identify specific loci associated. I will present our work using evolutionary analyses to generate a high-resolution picture of the emergence, global spread and local transmission of the pathogen as well as genomic determinants associated. I will show how non-selective processes like genetic drift contribute to the genomic diversity of the pathogen with downstream consequence at the transcription and methylation levels. In the second part of the talk I will also discuss different approaches to identify and track new drug resistance determinants using a combination of functional genomics and genomic diversity analyses from different countries and from different patients through time and space. Overall our analyses reveal new bacterial factors associated to virulence and drug resistance. I will show however how the frequency of genetic variants associated to different traits, even if advantageous, depend on the conditions of local TB control more than on the high fitness of the bacterial genotype.

\subsection{1}

doi: 10.15789/2220-7619-2018-4-6.11

\section{EMERGENCE OF BEDAQUILINE RESISTANCE AFTER COMPLETION OF BEDAQUILINE-BASED DRUG- RESISTANT TB TREATMENT: A CASE STUDY FROM SOUTH AFRICA}

M. de Vos ${ }^{1}$, S. Ley ${ }^{1}$, B. Derendinger ${ }^{1}$, A. Dippenaar ${ }^{1}$, M. Grobbelaar' ${ }^{1}$, A. Reuter ${ }^{2}$, J. Daniels ${ }^{2}$, S. Burns ${ }^{3}$, G. Theron ${ }^{1}$, J. Posey ${ }^{3}$, R. Warren ${ }^{1}$, H. Cox ${ }^{4}$

${ }^{1} D S T / N R F$ Centre of Excellence in Biomedical Tuberculosis Research/SAMRC Centre for Tuberculosis Research, Division of Molecular Biology and Human, Faculty of Medicine and Health Science, Stellenbosch University, South Africa; ${ }^{2}$ Médecins Sans Frontières, Operational Centre Brussels (OCB), Khayelitsha Project, Cape Town, South Africa: ${ }^{3}$ Division of Tuberculosis Elimination, National Center for HIV/AIDS, Viral Hepatitis, STD, and TB Prevention, Centers for Disease Control and Prevention, Atlanta, Georgia, United States; ${ }^{4}$ Institute of Infectious Disease and Molecular Medicine and Division of Medical Microbiology, Department of Pathology, Faculty of Health Sciences, University of Cape Town, South Africa

Treatment outcomes for drug-resistant tuberculosis (DR-TB) are poor with only $52 \%$ of MDR-TB and $24 \%$ of XDR-TB patients successfully treated. To address the global DR-TB epidemic WHO has released guidelines for the use of bedaquiline (BDQ) for the treatment of rifampicin-resistant or MDR-TB for specific indications. However, standardised methods to perform drug susceptibility testing (DST) have not been defined and BDQ resistance mechanisms remain poorly characterised.

Illumina NextSeq whole genome sequencing (WGS) was used to characterise serial Mycobacterium tuberculosis (Mtb) isolates from a patient receiving BDQ in Khayelitsha, South Africa. Phenotypic drug susceptibility testing (DST) for BDQ was performed in MGIT-960 media (concentration $1 \mu \mathrm{g} / \mathrm{ml}$ ).

WGS showed an initial infection with a strain resistant to 7 drugs (rifampicin, isoniazid (low-level), ethambutol, ethionamide, fluoroquinolones, pyrazinamide and streptomycin). Following initial treatment failure with a standardised MDR-TB regimen, the patient was placed on a regimen containing 6 effective drugs (including BDQ, based on WGS). Isolates taken prior to BDQ initiation were BDQ-susceptible (phenotypically). WGS of subsequent serial isolates revealed the acquisition of a variant in $R v 0678$ (conferring BDQ-resistance) one month after stopping BDQ treatment. Subsequent isolates showed the loss and gain of several other $R v 0678$ variants, with only one variant (138 $\mathrm{G}$ insertion) fixed in the last available isolate. All isolates with $R v 0678$ variants were BDQ-resistant.

The systematic gain and loss of $R v 0678$ variants in isolates taken after completion of BDQ-based treatment illustrates the complex ongoing evolution patterns of $M$. tuberculosis as the concentration of BDQ decreases in the patient (long half-life). An alternative explanation is the emergence of existing BDQ-resistant Mtb from lesions which rupture following continuation of treatment without BDQ and after stopping all TB treatment. The emergence of BDQ resistant $M$. tuberculosis following stopping of treatment poses a risk of transmission of BDQ resistant clones to close contacts. Monitoring of pre-existing and emerging BDQ resistance should be a priority for all routine use and should continue post BDQ cessation.

\subsection{2 doi: 10.15789/2220-7619-2018-4-6.12 \\ WHOLE GENOME SEQUENCING SHEDS LIGHT ON THE TRANSMISSION DYNAMICS OF A MULTI- DRUG RESISTANT MYCOBACTERIUM TUBERCULOSIS OUTBREAK OVER 23 YEARS IN A HIGH INCIDENCE SETTING}

A. Dippenaar ${ }^{1}$, R.M. Warren 1 , M. de $\operatorname{Vos}^{1}$, T. Heupink ${ }^{2}$, A. van Rie $^{2}$, C. Clarke', J. Posey ${ }^{3}$, S.L. Sampson', E.M. Streicher ${ }^{1}$

${ }^{1}$ DST-NRF Centre of Excellence for Biomedical Tuberculosis Research; South African Medical Research Council Centre for Tuberculosis Research; Division of Molecular Biology and Human Genetics, Faculty of Medicine and Health Sciences, Stellenbosch University, Cape Town, South Africa; ${ }^{2}$ Global Health Institute, Epidemiology and Social Medicine, Faculty of Medicine, University of Antwerp, Antwerp, Belgium; ${ }^{3}$ Centers of Disease Control and Prevention, Atlanta, GA, USA

Whole genome sequencing (WGS) has shown that Mycobacterium tuberculosis strains are more genetically diverse than previously assumed and that traditional genotyping methods cannot discriminate strain heterogeneity with high resolution, which may mask their ability to accurately define the directionality of an outbreak, particularly in high tuberculosis (TB) incidence settings.

The objective of this study was to examine the evolution of a single $M$. tuberculosis cluster defined by a particular IS6110 RFLP pattern to understand transmission and strain diversity over time.

Clinical M. tuberculosis isolates $(\mathrm{n}=97)$ with identical IS6110 RFLP fingerprint patterns were selected from a longitudinal sample bank of $M$. tuberculosis isolates collected from a high TB incidence suburb in the Western Cape, South Africa from 1993-2015. DNA was extracted from M. tuberculosis cultures for WGS and subsequent analysis. Available WGS of M. tuberculosis isolates from surrounding suburbs were screened and additional isolates 